\title{
Unilateral digital arterial ligation combined with low molecular weight heparins in severed finger without venous anastomosis
}

\author{
XUEMING CHEN, ZHENG CHEN, JIANDONG ZHOU and YAJUN XU
}

\author{
Department of Hand Surgery, Wuxi 9th Affiliated Hospital of Soochow University, Wuxi, Jiangsu 214000, P.R. China
}

Received October 21, 2017; Accepted May 15, 2018

DOI: $10.3892 /$ etm.2018.6174

\begin{abstract}
Curative effect of unilateral digital arterial ligation combined with low molecular weight heparins in the treatment of severed finger without venous anastomosis and its influence on venous crisis was studied. A total of 80 patients with distal severed finger treated in Wuxi No. 9 People's Hospital from May 2014 to July 2016 were selected into the study. According to the random number table, they were divided into the control group $(n=40)$ and the research group $(n=40)$. The patients in control group were treated with unilateral digital arterial ligation, while the patients in research group were treated with unilateral digital arterial ligation combined with low molecular weight heparin. The wound healing time after operation, hospitalization time, hemorheology detection results, survival rate and necrosis rate of replanted finger, incidence rate of venous crisis and recovery effect of replanted finger were compared between the two groups. After operation, the wound healing time and hospitalization time of patients in research group were lower than those in control group, and the differences were statistically significant $(\mathrm{p}<0.05)$. At $72 \mathrm{~h}$ after operation, the platelet adhesion rate, whole blood viscosity, whole blood low-shear viscosity, hematocrit and fibrinogen level in patients in research group were lower than those in control group, and the differences were statistically significant $(p<0.05)$. At 7 th day after operation, the survival rate of replanted finger in the research group was higher than that in control group, but the necrosis rate of replanted finger and incidence rate of venous crisis were lower than those in control group, and the differences were statistically significant $(\mathrm{p}<0.05)$. At 6 months after operation, the nail length, sensation, two-point discrimination and mobility of distal interphalangeal joint of patients in the research group were superior to those in control group, and the differences were statistically significant $(\mathrm{p}<0.05)$.
\end{abstract}

Correspondence to: Dr Yajun Xu, Department of Hand Surgery, Wuxi 9th Affiliated Hospital of Soochow University, 999 Liangxi Road, Binhu, Wuxi, Jiangsu 214000, P.R. China

E-mail: chenxm1980@qq.com

Key words: unilateral digital arterial ligation, low molecular weight heparin, severed finger without venous anastomosis, curative effect, venous crisis
Unilateral digital arterial ligation combined with low molecular weight heparin has a significant effect in the treatment of severed finger without venous anastomosis, which can effectively reduce or prevent the occurrence of venous crisis, improve the survival rate of replanted finger and promote the function recovery of replanted finger, so it is worthy of clinical promotion.

\section{Introduction}

In recent years, with the rapid development of social economy in China, the incidence rate of finger disability due to a variety of reasons has shown an increasing trend, seriously affecting the patient's labor ability and normal life, and increasing economic and psychological pressure on patients and their families (1). At present, the clinical treatment method of finger disability is mainly the early replantation, and with the progress and improvement of clinical micro-technique level, the survival rate of replanted finger has been significantly improved. Studies have found that the key to successful severed finger replantation is the connection of blood vessels. However, there is no appropriate venous anastomosis in the severed finger of patients with distal severed finger without venous anastomosis, so the survival rate after severed finger replantation is lower, and patients are prone to vascular crisis after operation (2). Unilateral digital arterial ligation, as a new surgical method, can effectively improve the survival rate of severed finger without venous anastomosis, but its specific mechanism of treatment is not fully clear yet in clinical medicine, and needs further study (3). Low-molecular weight heparin, as a kind of common anticoagulant, can prevent and treat venous thrombosis, and has been widely used in clinical treatment (4). On this basis, the severed finger without venous anastomosis was treated with unilateral digital arterial ligation combined with low molecular weight heparin in this study, and its clinical effect and influence on venous crisis were analyzed.

\section{Patients and methods}

Inclusion criteria. i) Patients diagnosed as severed finger without venous anastomosis via clinical examination; the severed finger refers to the abruption of arterial arch from the distal severed interphalangeal joint to the digital arterial arch; ii) patients without coagulation dysfunction; iii) patients 
without drug allergy history or allergic constitution; iv) patients without malignant tumor, nervous system diseases or endocrine system diseases (5); v) this study was approved by Ethics Committee of Wuxi No. 9 People's Hospital (Jiangsu, China); vi) all patients and their families were informed of this study, and signed the informed consent.

Exclusion criteria. i) Patients with severe organ diseases in heart, liver or kidney (6); ii) pregnant or lactating women; iii) patients allergic to anesthesia and low molecular weight heparin or who could not tolerate operation; and iv) patients who did not participate voluntarily.

Demographic data. A total of 80 patients with distal severed finger (98 fingers) treated in our hospital from May 2014 to July 2016 were selected as the subjects of study and they all conformed to the inclusion criteria. According to the random number table, they were divided into control group $(n=40)$ and research group $(n=40)$. In control group, there were 22 males ( 25 fingers) and 18 females (23 fingers) aged 20-60 years with an average age of $35.4 \pm 5.5$ years. In terms of injury cause, there were 4 cases of machine cutting injury, 19 cases of saw injury and 17 cases of machine runover injury. In research group, there were 24 males (28 fingers) and 16 females (22 fingers) aged 20-60 years with an average age of $35.9 \pm 4.7$ years. In terms of injury cause, there were 5 cases of machine cutting injury, 20 cases of saw injury and 15 cases of machine run-over injury. There were no statistically significant differences in the basic data between the two groups ( $p>0.05$ ), and the data were comparable.

Surgical procedures. Patients in both groups were treated with unilateral digital arterial ligation. Under the local infiltration anesthesia or brachial plexus block anesthesia, the operation was performed with hemostasis by compression using the pneumatic tourniquet on upper arm or rubber strip at the root of the finger. After the conventional cleaning of injured hand and severed fingers, the free necrotic tissue and foreign bodies on the two cross sections were carefully removed, followed by routine disinfection with $0.1 \%$ povidone iodine disinfectant and draping. The operator protected the nerves and blood vessels carefully under the microscope, completely removed the necrotic tissue and checked the proximal severed end. Moreover, the digital arteries and severed nerve ends were observed and marked, followed by the bandage and release of the tourniquet. After that, the distal severed end was checked using the same method, but no anastomotic vein could be detected; the digital arteries and severed nerve ends were also observed and marked. According to the vascular conditions, if necessary, the finger bone was appropriately shortened, the marrow cavity was penetrated using the Kirschner wire and the severed end was fixed. In the absence of tension, the tendon, nerves and blood vessels were anastomosed, and the wound was sutured. At the same time, the tourniquet and rubber strip at the root of finger were released, and the replanted finger became ruddy slowly. After the unilateral digital arterial ligation in the proximal end of replanted finger and wound dressing, the functional position of injured hand was fixed with plaster support. After operation, patients in the two groups received the anti-coagulation, anti-infection, anti-spasm and
Table I. Comparisons of wound healing time and hospitalization time after operation of patients between the two groups (mean $\pm \mathrm{SD}$, min).

\begin{tabular}{lcc}
\hline Groups & $\begin{array}{c}\text { Wound healing time } \\
\text { after operation }\end{array}$ & $\begin{array}{c}\text { Hospitalization time } \\
\text { after operation }\end{array}$ \\
\hline Control $(\mathrm{n}=40)$ & $8.7 \pm 2.3$ & $15.8 \pm 2.7$ \\
Research $(\mathrm{n}=40)$ & $6.3 \pm 1.4$ & $11.2 \pm 2.5$ \\
t-test & 5.637 & 7.906 \\
P-value & $0.000 ?$ & $0.000 ?$ \\
\hline
\end{tabular}

other symptomatic treatment. If the patient suffered from be finger darkening and swelling, cyanosis and venous return obstruction, the nails should be removed, and a longitudinal incision on nail bed and a side incision on fingertip were made to discharge appropriate amount of blood. The patients in the research group were injected subcutaneously with $0.4 \mathrm{ml}$ low molecular weight heparin (NMPN H20060191; Shenzhen Saibaoer Biopharmaceutical Co., Ltd., Shenzhen, China) 1 time/12 h for consecutive 3 days. Patients in the two groups were followed up for 6 months after operation.

Observation indexes. The wound healing time after operation, hospitalization time and hemorheology detection results were observed and compared between the two groups. The temperature of fingertip, the fullness of finger pulp, skin color and capillary response of patients were observed at 7 days after operation, thus determining the survival rate and necrosis rate of replanted finger and incidence rate of venous crisis after operation. At the same time, the nail length, sensory level, two-point discrimination (2-PD) and mobility of distal interphalangeal joint (DIPJ) of the replanted finger were evaluated according to the follow-up results. i) Venous crisis: The skin temperature of replanted finger was observed; if it declined suddenly, the finger turned from red to pale, the filling of capillaries in fingertip and nail disappeared, finger pulp shrunk, and there was no bleeding in the side incision of fingertip and dark purple blood sometimes slowly overflowed, and it was deemed as venous crisis (7). ii) Hemorheology: The platelet adhesion rate, whole blood viscosity, whole blood low-shear viscosity, hematocrit and fibrinogen, in patients were detected at $72 \mathrm{~h}$ after operation using a capillary viscometer (8).

Statistical analysis. Statistical Product and Service Solutions (SPSS, version X; SPSS, Inc., Chicago, IL, USA) 20.0 statistical software was used for data analysis. Enumeration data are presented as percentage and case (n, \%). Chi-square test was used for comparison of survival rate and necrosis rate. Measurement data are presented as mean $\pm \mathrm{SD}$, and t-test was used for intergroup comparison. $\mathrm{P}<0.05$ was considered to indicate a statistically significant difference.

\section{Results}

Wound healing time and hospitalization time after operation. After operation, the wound healing time and hospitalization time of patients in the research group were lower than those 
Table II. Comparison of hemorheology detection results of severed finger of patients between the two groups at $72 \mathrm{~h}$ after operation (mean $\pm \mathrm{SD})$.

\begin{tabular}{lccccc}
\hline Groups & $\begin{array}{c}\text { Platelet adhesion } \\
\text { rate }(\%)\end{array}$ & $\begin{array}{c}\text { Whole blood } \\
\text { viscosity }\left(15^{\text {s-1 }}\right)\end{array}$ & $\begin{array}{c}\text { Whole blood low-shear } \\
\text { viscosity }\left(150^{\text {s-1 }}\right)\end{array}$ & Hematocrit (\%) & Fibrinogen (g/l) \\
\hline Control $(\mathrm{n}=40)$ & $41.8 \pm 5.2$ & $8.4 \pm 0.2$ & $7.5 \pm 0.3$ & $47.1 \pm 4.2$ & $5.0 \pm 1.5$ \\
Research $(\mathrm{n}=40)$ & $32.8 \pm 2.7$ & $7.8 \pm 0.1$ & $6.3 \pm 0.2$ & $31.6 \pm 3.3$ & $3.1 \pm 1.0$ \\
t-test & 9.715 & 16.971 & 21.049 & 18.353 & 6.666 \\
P-value & $<0.000$ & $<0.000$ & $<0.000$ & $<0.000$ & $<0.000$ \\
\hline
\end{tabular}

Table III. Comparison of survival rate and necrosis rate of replanted finger, and incidence rate of venous crisis of patients between the two groups at 7 days after operation (n, \%).

\begin{tabular}{lccc}
\hline Groups & $\begin{array}{c}\text { Survival of } \\
\text { replanted finger }\end{array}$ & $\begin{array}{c}\text { Necrosis of } \\
\text { replanted finger }\end{array}$ & $\begin{array}{c}\text { Venous } \\
\text { crisis }\end{array}$ \\
\hline Control $(\mathrm{n}=40)$ & $30(75.0)$ & $10(25.0)$ & $12(30.0)$ \\
Research $(\mathrm{n}=40)$ & $37(92.5)$ & $3(7.5)$ & $3(7.5)$ \\
$\chi^{2}$ & 4.501 & 4.501 & 6.951 \\
P-value & 0.034 & 0.034 & 0.008 \\
\hline
\end{tabular}

Table IV. Comparison of recovery effects of replanted finger between the two groups at 6 months after operation $($ mean $\pm S D)$.

\begin{tabular}{lcccc}
\hline Groups & Nail length & Sensation & 2-PD & $\begin{array}{c}\text { Mobility of } \\
\text { DIPJ }\end{array}$ \\
\hline Control $(\mathrm{n}=40)$ & $11.3 \pm 0.8$ & $2.5 \pm 0.2$ & $7.5 \pm 0.4$ & $44.8 \pm 3.2$ \\
Research $(\mathrm{n}=40)$ & $15.8 \pm 4.3$ & $3.7 \pm 0.4$ & $5.0 \pm 0.7$ & $59.7 \pm 5.3$ \\
t-test & 6.507 & 16.971 & 19.612 & 15.221 \\
P-value & $0.000 ?$ & $0.000 ?$ & $0.000 ?$ & $0.000 ?$ \\
\hline
\end{tabular}

2-PD, two-point discrimination; DIPJ, distal interphalangeal joint.

in control group, and the differences were statistically significant $(\mathrm{p}<0.05)$ (Table I).

Hemorheology detection results. At $72 \mathrm{~h}$ after operation, the platelet adhesion rate, whole blood viscosity, whole blood low-shear viscosity, hematocrit and fibrinogen level in patients in research group were lower than those in control group, and the differences were statistically significant $(\mathrm{p}<0.05)$ (Table II).

Recovery condition of replanted finger. At 7 days after operation, the survival rate of replanted finger in the research group was higher than that in control group, but the necrosis rate of replanted finger and incidence rate of venous crisis were lower than those in control group, and the differences were statistically significant $(\mathrm{p}<0.05)$ (Table III).

Functional recovery effect of replanted finger. At 6 months after operation, the nail length, sensation, 2-PD and mobility of DIPJ of patients in the research group were superior to

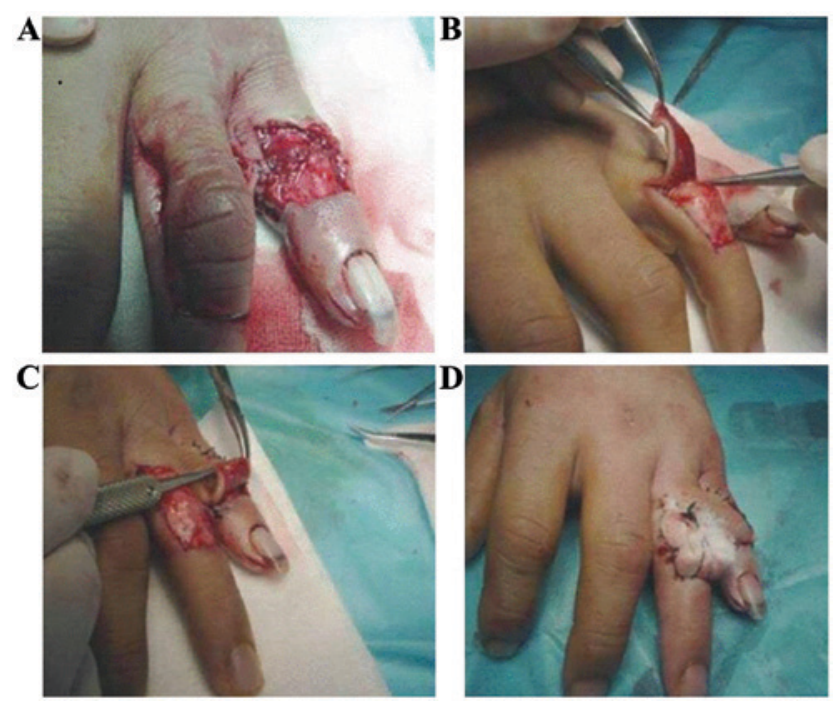

Figure 1. Surgical procedures for unilateral digital arterial ligation. (A) Remove free necrotic tissue and clean up the wound; (B) prepare the flap; (C) transfer the flap; and (D) graft free flap and suture the wound edge.
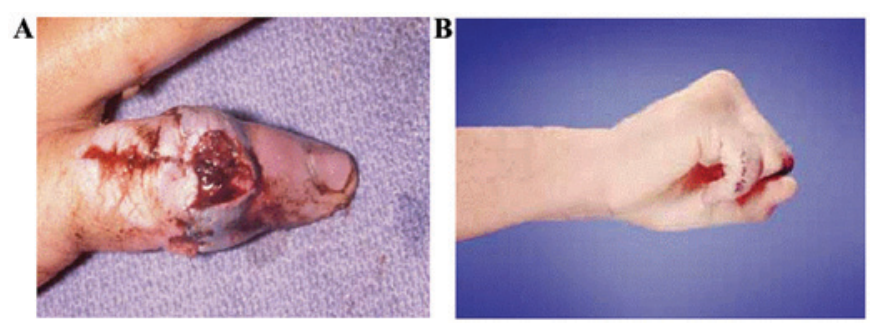

Figure 2. Images before operation (A) and (B) after surgery.

those in control group, and the differences were statistically significant $(\mathrm{p}<0.05)($ Table IV).

Surgical procedure and recovery condition of severed finger without venous anastomosis. Unilateral digital arterial ligation does not reduce the arterial blood supply of fingers in the treatment of severed finger without venous anastomosis, which is characterized by high survival rate and good postoperative recovery (Figs. 1 and 2).

\section{Discussion}

With the rapid development and perfection of microsurgical technique, gradual maturity of small vascular anastomosis 
technique in clinical medicine and the increasingly higher requirement of people for modern life quality, people have had higher and higher requirements of severed finger replantation due to the serious impact of severed finger on the appearance, function, psychology and normal communication of patients $(9,10)$. Therefore, it was found in clinical medicine on the basis of body anatomy that there are direct communicating branches between the branch and vein of dorsal digital artery, and it is difficult for the corresponding supply of finger blood to return through the vein when the physiological reflux veins disappear (11); but the proximal digital arterial ligation after one-side anastomosis can reduce the arterial blood perfusion of the replanted finger to a certain extent, buffer the venous return load, and form a new blood circulation from the dorsal digital arterial branches to the dorsal digital veins (12).

Studies have shown that in the bilateral digital arterial anastomosis in the unilateral digital arterial ligation, the digital arterial section was ligated at $2-4 \mathrm{~cm}$, so that the venous return of replanted finger can be promoted to shunt towards the dorsal digital veins via the dorsal digital arterial branches, thus completing the new blood circulation. In early stage, new collateral venous return can be formed at 3-7 days after the low-level blood supply, promoting the survival of severed fingers $(13,14)$. Therefore, it is speculated that no venous return is found in the severed finger after the connection of bilateral arteries, so the incidence rate of venous crisis after operation gradually increases under the sufficient arterial supply but no venous return. There are distal arterial arches in the digital arteries, combined with a large number of branches, so the simple ligation of one-side artery has no significant threat to the blood supply of finger tips, but can effectively reduce the pressure of relevant tissue in finger tips after the replantation of the severed finger; and the blood return can fully flow back to veins via collateral circulation of non-severed end, promoting the blood circulation (15). But we need to pay attention in the unilateral digital arterial ligation: i) The surgical operation should be gentle to avoid damage to the blood vessels; ii) whether patients meet the surgical indications should be assessed before surgery (16); iii) the non-invasive and micro-operation principles should be followed; iv) in the arterial anastomosis under the microscope, thicker blood vessels should be anastomosed furthest, the operation should be gentle, and the anastomotic blood vessels should be smooth enough; and v) the digital arterial ligation should be implemented gently, the integrity of soft tissue around the ligated arteries should be guaranteed to the utmost and the digital arteries should be ligated in case of good blood supply in the severed end.

Venous crisis as a kind of common clinical complication, which is related to the poor quality of anastomosis, incomplete debridement in injured veins and postoperative tissue edema; if the patients do not receive timely treatment after venous crisis, it will be detrimental to postoperative recovery (17).

Low molecular weight heparin, as a kind of commonly-used anticoagulant, can effectively inhibit the thrombin and coagulation factor $\mathrm{Xa}$, and it can reach the peak in plasma at $3 \mathrm{~h}$ after subcutaneous injection and then decline, its half-life is $\sim 3.5 \mathrm{~h}$ (18); at the same time, a certain anticoagulant effect can be detected at $24 \mathrm{~h}$ after medication, and the activity of anticoagulant factor IIa in the course of medication is lower than that of anticoagulant factor Xa. Low molecular weight heparin is metabolized a little and removed in prototype via liver, and its half-life in the elderly patients is slightly extended (19). Studies have shown that the activity of anticoagulant factor Xa of low molecular weight heparin is significant, and the anticoagulant factor IIa and antithrombin activity is lower, so the low molecular weight heparin can effectively inhibit the in vivo and in vitro thrombosis and arteriovenous thrombosis in patients, nd has no significant effect on the platelet aggregation, fibrinogen and platelet binding and can play the anti-thrombus effect without higher risk of hemorrhage (20).

The results of this study showed that the healing time and hospitalization time of patients in the research group after treatment were lower than those in control group, the stability of hemorheology was higher than that in control group, the recovery effect of replanted finger after operation was superior to that of control group, and the incidence rate of venous crisis was lower than that in control group, indicating that unilateral digital arterial ligation combined with low molecular weight heparin has a significant effect in the treatment of severed finger without venous anastomosis, which can reduce the risk of venous crisis and improve the recovery of replanted finger. Lee et al study (21) showed that controlled systemic continuous heparinization improved the replantation success rate of artery-only anastomosis replantation in single distal digit amputation, indicated that the use of the anticoagulation is a safe and effective method in severed finger replantation, which coincide with our result. Compared with systemic continuous heparinization, low molecular weight heparin is safer, more effective, more durable and more stable. However, the sample size in this study was small, it was not a double-blind study, the observation time was shorter, and the safety of patients after surgery and medication was not analyzed. In addition, this was a single-center clinical study. To get more valid and credible results, the sample size can be expanded and a randomized, double-blind, placebo controlled, multicenter study should be conducted for in-depth study later.

In conclusion, unilateral digital arterial ligation combined with low molecular weight heparin has a significant effect in the treatment of severed finger without venous anastomosis, which can effectively reduce or prevent the occurrence of venous crisis, shorten the hospitalization time of patients, improve the survival rate of replanted finger and promote the function recovery of replanted finger, so it is worthy of clinical promotion.

\section{Acknowledgements}

Not applicable.

\section{Funding}

No funding was received.

\section{Availability of data and materials}

All data generated or analyzed during this study are included in this published article. 


\section{Authors' contributions}

$\mathrm{XC}$ designed the study, $\mathrm{ZC}$ was for data collection, $\mathrm{XC}$ and $\mathrm{YX}$ were for data analysis, ZC and JZ did the follow-up, XC and YX prepared the manuscript. All authors read and approved the final manuscript.

\section{Ethics approval and consent to participate}

This study was approved by the Ethics Committee of Wuxi No. 9 People's Hospital (Jiangsu, China). Signed informed consents were obtained from the patients and/or guardians.

\section{Consent for publication}

Patients or their guardians provided written informed consents for publication.

\section{Competing interests}

The authors declare that they have no competing interests.

\section{References}

1. Lee BI, Chung HY, Kim WK, Kim SW and Dhong ES: The effects of the number and ratio of repaired arteries and veins on the survival rate in digital replantation. Ann Plast Surg 44: 288-294, 2000.

2. Doi K, Hattori Y, Ikeda K and Dhawan V: Significance of shoulder function in the reconstruction of prehension with double free-muscle transfer after complete paralysis of the brachial plexus. Plast Reconstr Surg 112: 1596-1603, 2003.

3. Hasuo T, Nishi G, Tsuchiya D and Otsuka T: Fingertip replantations: Importance of venous anastomosis and the clinical results. Hand Surg 14: 1-6, 2009.

4. Koshima I, Yamashita S, Sugiyama N, Ushio S, Tsutsui T and Nanba Y: Successful delayed venous drainage in 16 consecutive distal phalangeal replantations. Plast Reconstr Surg 115: 149-154, 2005.

5. Hsu CC, Lin YT, Moran SL, Lin CH, Wei FC and Lin CH: Arterial and venous revascularization with bifurcation of a single central artery: A reliable strategy for Tamai Zone I replantation. Plast Reconstr Surg 126: 2043-2051, 2010.

6. Gustafsson M and Ahlström G: Emotional distress and coping in the early stage of recovery following acute traumatic hand injury: A questionnaire survey. Int J Nurs Stud 43: 557-565, 2006.

7. Whitaker IS, Josty IC, Hawkins S, Azzopardi E, Naderi N, Graf J, Damaris L, Lineaweaver WC and Kon M: Medicinal leeches and the microsurgeon: A four-year study, clinical series and risk benefit review. Microsurgery 31: 281-287, 2011.

8. van Alphen NA, Gonzalez A, McKenna MC, McKenna TK, Carlsen BT and Moran SL: Ciprofloxacin-resistant Aeromonas infection following leech therapy for digit replantation: Report of 2 cases. J Hand Surg Am 39: 499-502, 2014.
9. Zhao J, Abdullah S, Li WJ, Appukuttan A and Tien HY: A novel solution for venous congestion following digital replantation: A proximally based cross-finger flap. J Hand Surg Am 36: 1224-1230, 2011.

10. Zhang GL, Chen KM, Zhang JH and Wang SY: Hand reconstruction using heterotopic replantation of amputated index and little fingers. Chin J Traumatol 14: 316-318, 2011.

11. Yabe T, Tsuda T, Hirose S and Ozawa T: Treatment of fingertip amputation: Comparison of results between microsurgical replantation and pocket principle. J Reconstr Microsurg 28: 221-226, 2012.

12. McClain DA, Abuelgasim KA, Nouraie M, Salomon-Andonie J, Niu X, Miasnikova G, Polyakova LA, Sergueeva A, Okhotin DJ, Cherqaoui R, et al: Decreased serum glucose and glycosylated hemoglobin levels in patients with Chuvash polycythemia: A role for HIF in glucose metabolism. J Mol Med (Berl) 91: 59-67, 2013.

13. Martini M, Teofili L, Cenci T, Giona F, Torti L, Rea M, Foà R, Leone $G$ and Larocca LM: A novel heterozygous HIF2A ${ }^{\text {M535I }}$ mutation reinforces the role of oxygen sensing pathway disturbances in the pathogenesis of familial erythrocytosis. Haematologica 93: 1068-1071, 2008.

14. Richalet JP, Rivera-Ch M, Maignan M, Privat C, Pham I, Macarlupu JL, Petitjean O and León-Velarde F: Acetazolamide for Monge's disease: Efficiency and tolerance of 6-month treatment. Am J Respir Crit Care Med 177: 1370-1376, 2008.

15. Delton-Vandenbroucke I, Grammas P and Anderson RE: Regulation of n-3 and n- 6 fatty acid metabolism in retinal and cerebral microvascular endothelial cells by high glucose. J Neurochem 70: 841-849, 1998

16. Tang YB, Simchon S and Chen HC: Microcirculation of a venous flap: An experimental study with microspheres in rabbits. Scand J Plast Reconstr Surg Hand Surg 34: 207-212, 2000.

17. Wang L, Fu J, Li M, Han D and Yang L: Repair of hand defects by transfer of free tissue flaps from toes. Arch Orthop Trauma Surg 133: 141-146, 2013

18. Siminerio L, Ruppert KM and Gabbay RA: Who can provide diabetes self-management support in primary care? Findings from a randomized controlled trial. Diabetes Educ 39: 705-713, 2013.

19. Beacham BL and Deatrick JA: Health care autonomy in children with chronic conditions: Implications for self-care and family management. Nurs Clin North Am 48: 305-317, 2013.

20. Cicutto L, Brooks D and Henderson K: Self-care issues from the perspective of individuals with chronic obstructive pulmonary disease. Patient Educ Couns 55: 168-176, 2004.

21. Lee JY, Kim HS, Heo ST, Kwon H and Jung SN: Controlled continuous systemic heparinization increases success rate of artery-only anastomosis replantation in single distal digit amputation: A retrospective cohort study. Medicine (Baltimore) 95: e3979, 2016.

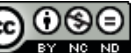

This work is licensed under a Creative Commons Attribution-NonCommercial-NoDerivatives 4.0 International (CC BY-NC-ND 4.0) License. 